$04 ; 13$

\title{
Энерговыделение при бомбардировке атомами дейтерия поверхности вольфрама
}

\author{
( Д.С. Мелузова, П.Ю. Бабенко, М.И. Миронов, В.С. Михайлов, А.П. Шергин, А.Н. Зиновьев \\ Физико-технический институт им. А.Ф. Иоффе РАН, Санкт-Петербург, Россия \\ E-mail: dmeluzova@gmail.com
}

Поступило в Редакцию 6 марта 2019г.

В окончательной редакции 6 марта 2019 г.

Принято к публикации 19 марта 2019г.

\begin{abstract}
Рассчитано распределение энерговыделения (линейных потерь энергии) по глубине при бомбардировке атомами дейтерия мишени из вольфрама в широком диапазоне энергий налетающих частиц $100 \mathrm{eV}-10 \mathrm{MeV}$. Показано, что в диапазоне энергий до $100 \mathrm{keV}$ максимум энерговыделения вопреки сложившимся представлениям находится вблизи поверхности твердого тела. При энергии свыше $100 \mathrm{keV}$ характер распределения меняется и появляется брэгговский максимум вблизи точки остановки частицы. Получено распределение энерговыделения по глубине в вольфраме для условий, типичных для токамака-реактора ИТЭР, что позволяет оценить нагрев стенки при бомбардировке атомами плазмы.
\end{abstract}

Ключевые слова: энерговыделение, линейные потери энергии, вольфрам, брэгговский максимум, токамакреактор.

DOI: 10.21883/PJTF.2019.11.47827.17771

В 1903 г. У. Брэгг установил, что при торможении альфа-частиц в воздухе зависимость потери энергии от глубины проникновения имеет выраженный пик (названный пиком Брэгга) вблизи точки остановки частицы. Основные потери энергии при торможении частиц связаны с ионизацией атомов мишени. Сечение этого процесса растет с уменьшением энергии частицы. Поэтому частица теряет значительную часть энергии вблизи точки остановки. Это обстоятельство используется в протонной терапии злокачественных опухолей $[1,2]$. Как следствие, основная доза сосредоточена в пораженной ткани при минимальном облучении здоровых клеток, расположенных ближе к поверхности.

В токамаке-реакторе ИТЭР планируется работа с дейтериво-тритиевой плазмой. В качестве основного материала дивертора, где будет происходить существенное энерговыделение, рассматривается вольфрам. Характерные энергии частиц плазмы будут лежать в диапазоне $100 \mathrm{eV}-20 \mathrm{keV}$, т.е. значительно ниже энергий частиц, применяемых в ионной терапии. Работы, в которых анализируется распределение энерговыделения по глубине для указанных условий, отсутствуют. Поэтому были выбраны вольфрам в качестве объекта и диапазон энергий частиц $100 \mathrm{eV}-10 \mathrm{MeV}$, чтобы проследить появление пика Брэгга.

Распределение энерговыделения по глубине связано с расчетом пробегов частиц в твердом теле. Обзор методов расчета и экспериментальных данных о пробегах и потерях энергии можно найти в $[3,4]$. Наиболее широко применяется код SRIM [5], в котором используется потенциал ZBL для описания рассеяния частиц. К сожалению, код SRIM напрямую не позволяет рассчитать распределение энерговыделения по глубине.
В приближении парных соударений (ППС) [3] траектория бомбардирующей частицы заменяется асимптотами траектории при сближении с атомом мишени и разлете частиц. В наших работах [6,7] проведено сравнение результатов расчетов коэффициентов отражения атомов водорода и дейтерия от поверхности вольфрама, получаемых при использовании метода ППС и более совершенного метода - метода расчета траекторий частиц. Было показано, что для рассматриваемого диапазона энергий оба метода дают близкие результаты. Поскольку использование ППС значительно ускоряет вычисления, в настоящей работе для расчета распределения линейных потерь энергии и пробегов по глубине материала мы использовали его модернизированную версию, описанную в работах [6,7].

На результаты вычислений серьезно влияет потенциал взаимодействия, применяемый для описания рассеяния частиц. На наш взгляд, наиболее точным является потенциал DFT [8], полученный в рамках приближения функционала плотности с использованием пакета Dmol для выбора базиса волновых функций. В работе [8] расчеты, выполненные с помощью данного метода, оказались в хорошем согласии с результатами, полученными из экспериментов по рассеянию частиц.

Для конкретного случая $\mathrm{D}-\mathrm{W}$ отталкивательная часть потенциала хорошо согласуется с результатами, полученными по формуле из работы [9], при этом потенциал спадает значительно быстрее с ростом межъядерного расстояния, чем потенциал ZBL. Другое отличие потенциал DFT предсказывает наличие притягивающей ямы в потенциале с глубиной $4.6 \mathrm{eV}$, что находится в согласии с независимыми расчетами [10]. Наличие потенциальной ямы влияет на траектории частиц и 


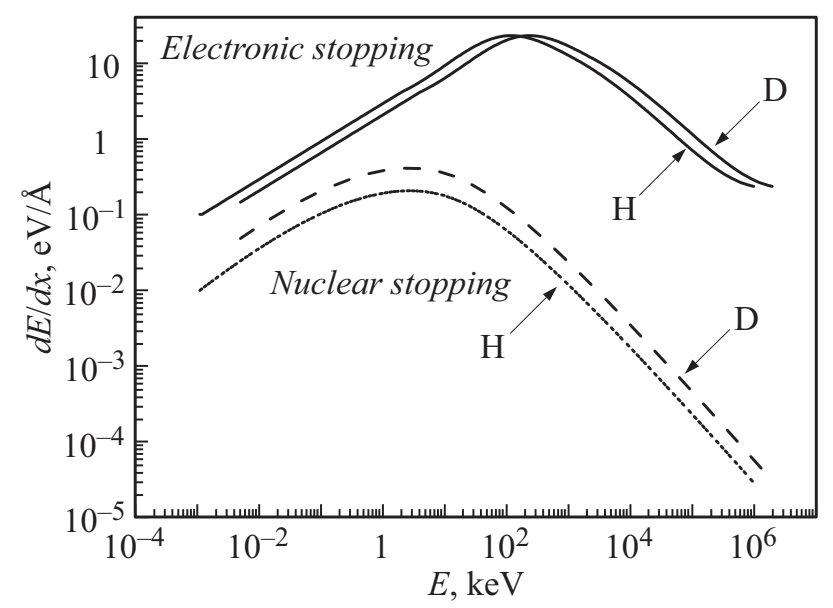

Рис. 1. Потери энергии при торможении на электронах и ядрах водорода и дейтерия в вольфраме. Использованы значения из базы данных [4].

существенно меняет результаты при низких начальных энергиях.

На рис. 1 представлены данные об электронных тормозных способностях, взятые из базы данных [4]. Для удобства расчетов эти данные были описаны следующими аналитическими зависимостями:

в диапазоне начальных энергий $E=1 \mathrm{eV}-25 \mathrm{keV}$

$$
\frac{d E}{d x}=2.858 E^{0.49}(1+0.004 E)
$$

в диапазоне $E=25-1000 \mathrm{keV}$

$$
\begin{aligned}
\frac{d E}{d x} & =\exp \left[1.936-0.853 \ln E+0.670 \ln ^{2} E\right. \\
& \left.-0.125 \ln ^{3} E+0.007 \ln ^{4} E\right]
\end{aligned}
$$

в диапазоне $E=1-10 \mathrm{MeV}$

$$
\frac{d E}{d x}=\frac{2231.3 \ln (E / 117.1)}{E^{0.86}}
$$

для $E=10-200 \mathrm{MeV}$

$$
\frac{d E}{d x}=\exp [9.04-0.97 \ln (\ln E)-0.61 \ln E] .
$$

В формулах начальная энергия $E$ задается в $\mathrm{keV}$, a величины $\mathrm{d} E / d x$ выражены в $\mathrm{eV} / \AA$.

Следует отметить, что электронные тормозные способности для различных изотопов водорода совпадают при равной скорости соударения. На рис. 1 для сопоставления приведены также данные для ядерных тормозных способностей систем $\mathrm{H}-\mathrm{W}$ и $\mathrm{D}-\mathrm{W}$. Ядерные тормозные способности возрастают пропорционально массе налетающей частицы. Как видно из рис. 1, в нашем случае электронные тормозные способности существенно превышают ядерные. Ядерные тормозные способности зависят от используемого потенциала. В наших расчетах они учитывались непосредственно при рассмотрении кинематики конкретного столкновения, а угол рассеяния рассчитывался с использованием потенциала DFT.

Для описания распределения энерговыделения по глубине часто используется термин ,линейные потери энергии“ $d W / d x[1]$, которые определяются как средние потери энергии частицей на интервале $d x$ (в наших расчетах величина $d x$ составляла $1 \AA$, а результаты усреднялись для $5 \cdot 10^{5}$ рассмотренных траекторий). Предполагается, что направление пучка и ось $x$ перпендикулярны поверхности.

Результаты расчетов энерговыделения (линейных потерь энергии) $d W / d x$ по глубине материала представлены на рис. 2. Величина $d W / d x$ включает потери энергии при торможении на электронах и ядрах. Вследствие многократного рассеяния величина проецированного на ось $x$ пробега меньше полного пробега частиц, поэтому величина $d W / d x$ больше суммы потерь энергии на электронах и ядрах.

Как следует из рис. 2, а, при энергиях ниже $100 \mathrm{keV}$ характер распределения отличен от традиционных пред-
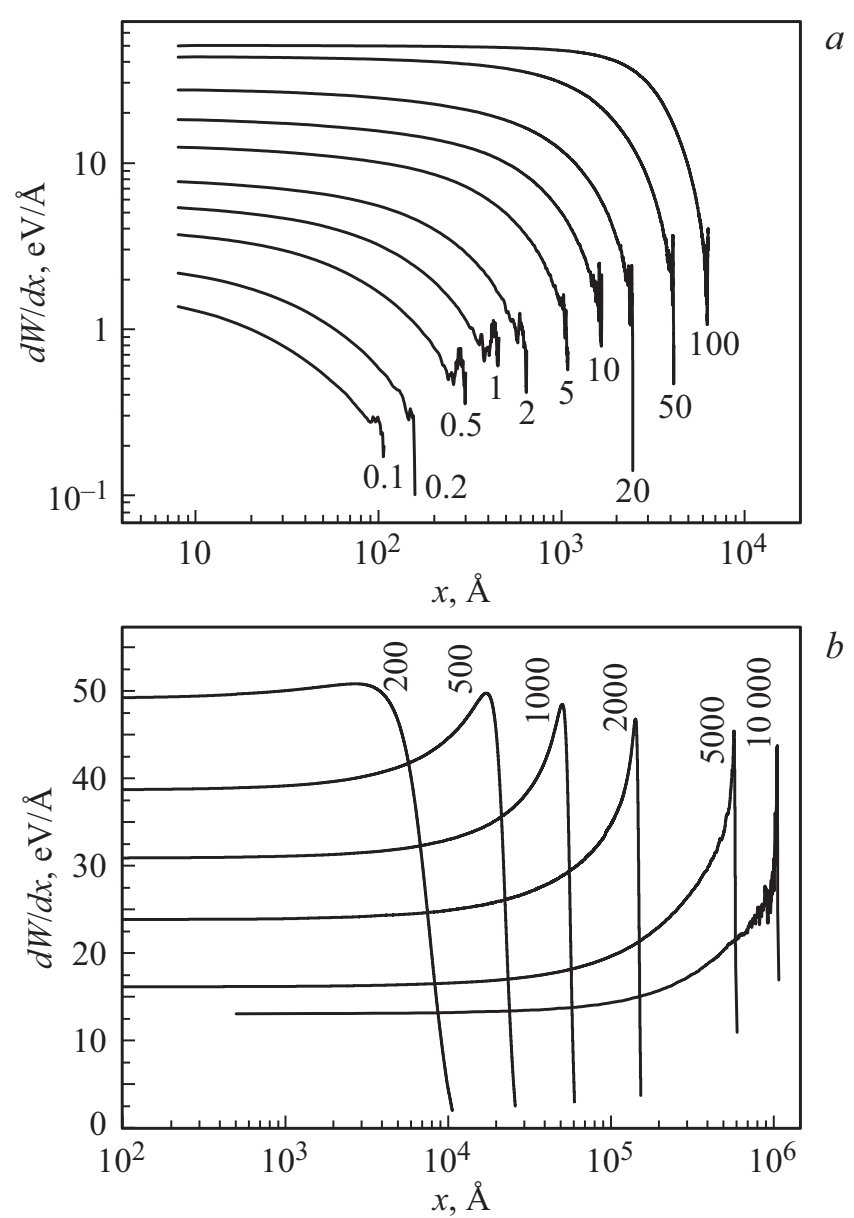

Рис. 2. Энерговыделение (линейные потери энергии) по глубине материала для ионов водорода при бомбардировке вольфрама. Числа около кривых - начальная энергия (в $\mathrm{keV})$. $a$ - диапазон энергий $0.1-100 \mathrm{keV}, b-$ диапазон энергий $200-10000 \mathrm{keV}$. 

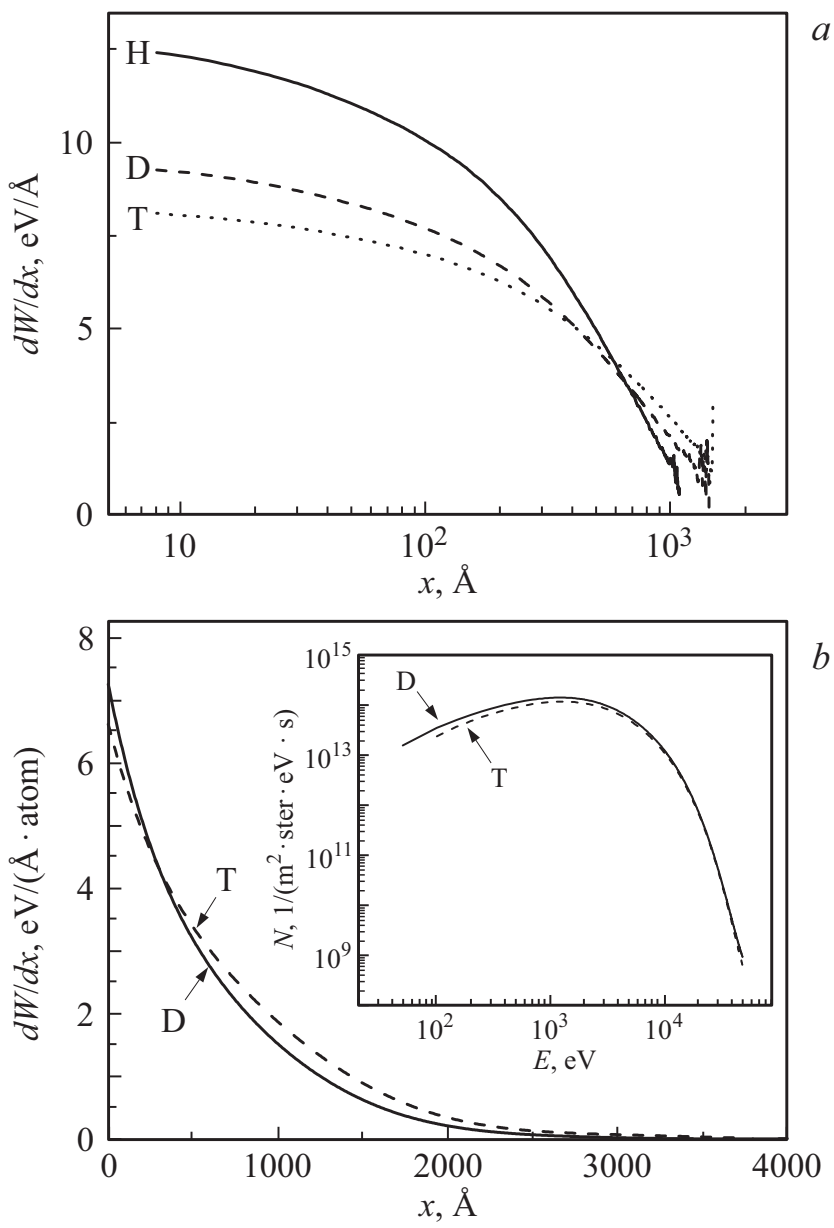

Рис. 3. $a-$ энерговыделение (линейные потери энергии) по глубине материала для различных изотопов водорода при начальной энергии $5 \mathrm{keV}, b-$ нормированное на одну падающую частицу распределение энерговыделения по глубине в вольфраме, облучаемом типичным для токамака ИТЭР спектром атомов дейтерия и трития, покидающих плазму [11] (показан на вставке).

ставлений. Максимум энерговыделения наблюдается вблизи поверхности, и с ростом глубины происходит спад энерговыделения. Это связано с тем, что при энергиях до $100 \mathrm{keV}$ сечение тормозных потерь на электронах растет с ростом энергии частицы. При энергиях порядка $100 \mathrm{keV}$ распределение по глубине уплощается, а при дальнейшем росте начальной энергии в распределении энергии по глубине появляется брэгговский пик вблизи точки остановки частиц (рис. $2, b$ ). Следует отметить, что контрастность брэгговского пика растет с ростом энергии. При энергии $5 \mathrm{MeV}$ контрастность пика не превышает 2, значительное энерговыделение происходит в приповерхностных слоях, и это должно учитываться в медицинских приложениях.

Результаты расчетов для случая столкновений ионов различных изотопов водорода с вольфрамом представлены на рис. $3, a$ для энергии $5 \mathrm{keV}$ - ожидаемой средней энергии частиц в токамаке ИТЭР. Поведение $d W(x) / d x$ для изотопов водорода подобно. При малых $x$ величина $d W / d x$ больше для водорода, что связано с бо́льшим значением электронных тормозных способностей при одинаковой энергии. С этим же связано и то, что проективный пробег водорода при одной и той же начальной энергии меньше, чем для дейтерия и трития.

В плазме токамака ИТЭР при работе на смеси дейтерия и трития вследствие процессов перезарядки и фоторекомбинации будут образовываться атомы, покидающие плазму. Типичный энергетический спектр атомов дейтерия и трития $d N / d E$, бомбардирующих первую стенку токамака, был рассчитан в работе [11] (см. вставку на рис. $3, b)$. Для такого энергетического спектра мы рассчитали зависимость энерговыделения от глубины, нормируя результат на одну падающую частицу (рис. $3, b$ ). Отражение частиц от стенки было учтено нами при расчете величин $d W / d x$. Как и в случае моноэнергетического пучка, максимум энерговыделения наблюдается вблизи поверхности, а характерная глубина связана со средней энергией частиц в спектре падающих атомов. Атомы трития в среднем проникают глубже в материал стенки по сравнению с атомами дейтерия, что может приводить к накоплению трития в первой стенке токамака-реактора.

Сформулируем основные выводы.

1. Рассчитано распределение энерговыделения по глубине материала при бомбардировке вольфрама протонами в широком диапазоне начальных энергий.

2. Показано резкое различие распределений энерговыделения для разных диапазонов начальных энергий: для энергий менее $100 \mathrm{keV}$ максимальное энерговыделение происходит вблизи поверхности, для энергий свыше $200 \mathrm{keV}$ наблюдается брэгговский пик. Это объясняется различием в энергетической зависимости электронных тормозных способностей: при энергиях ниже $100 \mathrm{keV}$ тормозная способность растет, а при энергиях свыше $100 \mathrm{keV}$ падает с ростом начальной энергии частицы.

3. Рассчитано распределение энерговыделения по глубине для условий, типичных для токамака-реактора ИТЭР, что позволяет оценить нагрев приповерхностных слоев при бомбардировке стенки атомами, покидающими плазму. Предсказывается, что при бомбардировке поверхности атомами дейтерия и трития при ожидаемом одинаковом спектре частиц по энергии атомы трития будут имплантироваться глубже, чем атомы дейтерия, что может вызвать накопление трития в первой стенке токамака-реактора.

4. Контрастность брэгговского максимума при $E<10 \mathrm{MeV}$ невелика, и наличие заметного энерговыделения вне пика должно учитываться в медицинских исследованиях.

\section{Финансирование работы}

Работа выполнена в рамках государственного задания Министерства образования и науки РФ для Федерального государственного бюджетного учреждения 
науки Физико-технического института им. А.Ф. Иоффе Российской академии наук.

\section{Конфликт интересов}

Авторы заявляют, что у них нет конфликта интересов.

\section{Список литературы}

[1] Кленов Г.И., Хорошков В.С. // УФН. 2016. Т. 186. № 8. C. 891-911. DOI: 10.3367/UFNr.2016.06.037823

[2] Durante M., Paganetti H. // Rep. Prog. Phys. 2016. V. 79. N 9. P. 096502. DOI:10.1088/0034-4885/79/9/096702

[3] Экштайн B. Компьютерное моделирование взаимодействия частиц с поверхностью твердого тела. М.: Мир, 1995. $321 \mathrm{c}$.

[4] Paul H. IAEA NDS. https://www-nds.iaea.org/stopping

[5] Ziegler J.F., Biersack J.P. SRIM. http://www.srim.org

[6] Бабенко П.Ю., Зиновьев А.Н., Мелузова Д.С., Шергин А.П. // Поверхность. Рентгеновские, синхротронные и нейтронные исследования. 2018. № 6. С. 7-12. DOI: $10.7868 / \mathrm{S} 0207352818060021$

[7] Babenko P.Yu., Deviatkov A.M., Meluzova D.S., Shergin A.P., Zinoviev A.N. // Nucl. Instrum. Meth. Phys. Res. B. 2017. V. 406. P. 538-542. DOI: 10.1016/j.nimb.2016.12.043

[8] Zinoviev A.N., Nordlund K. // Nucl. Instrum. Meth. Phys. Res. B. 2017. V. 406. P. 511-517. DOI: 10.1016/j.nimb.2017.03.047

[9] Zinoviev A.N. // Nucl. Instrum. Meth. Phys. Res. B. 2011. V. 269. N 9. P. 829-833. DOI: 10.1016/j.nimb.2010.11.074

[10] Anders L.W., Hansen R.S., Bartell L.S. // J. Chem. Phys. 1973. V. 59. N 10. P. 5277-5287. DOI: $10.1063 / 1.1679870$

[11] Afanasyev V.I., Chernyshev F.V., Kislyakov A.I., Kozlovski S.S., Lyublin B.V., Mironov M.I., Melnik A.D., Nesenevich V.G., Petrov M.P., Petrov S.Ya. // Nucl. Instrum. Meth. Phys. Res. A. 2010. V. 621. N 1-3. P. 456-467.

DOI: $10.1016 /$ j.nima.2010.06.201 